\title{
Found in translation
}

\section{For those who wish to marry research with clinical applications, finding the right environment can be hard.}

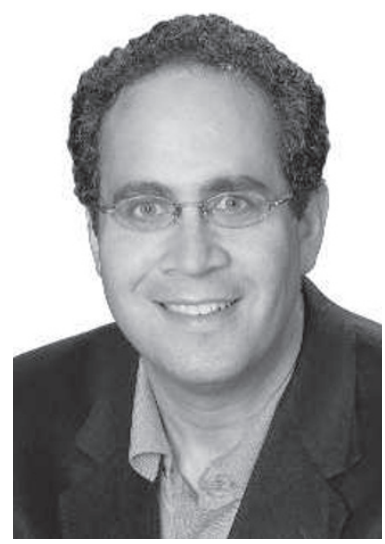

David Shaywitz

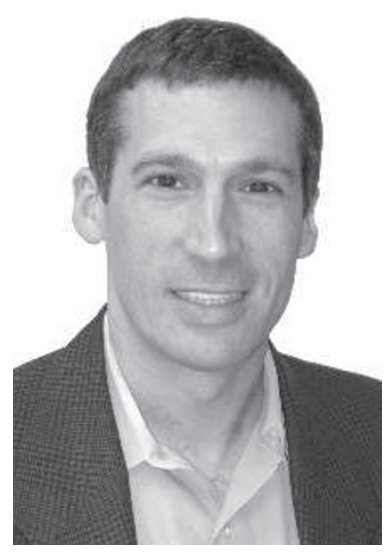

Hayes Dansky
The appeal of the career path as a physician-scientist is perhaps matched only by the challenge of the career itself. Many trainees ultimately discover that the elegant concept of uniting the rigour of basic science with the compassion of patient care can be remarkably unwieldy in practice.

The need to develop - and support - translational researchers is acknowledged by leading academic medical centres, as well as by the roadmap set out by the US National Institutes of Health (NIH). "It is more and more difficult to recruit, mentor, train and retain a critical mass of clinical and translational scientists," NIH director Elias Zerhouni wrote in 2005, adding: "The increasingly complex resources needed to conduct modern clinical and translational research are either missing or scattered."

Zerhouni's description rings true. In our academic medical centres, those primarily interested in basic research or clinical care can readily find exceptional role models and well-defined career paths. Yet the aspiring translational researcher often feels cast adrift, wondering when the long-anticipated integration of science and medicine is finally going to happen.

As much as we enjoyed the basic science with which we were engaged, our determination to pursue translational research led both of us, very early in our careers, to leave attractive, well-funded academic positions to join the newly formed Department of Experimental Medicine at Merck Research Labs. On balance, our department has grown less by a formal recruiting process, and more by the self-selection of likeminded physician-scientists and colleagues who share a passion for turning promising science into clinical application.

In our experimental medicine division, we design early-stage clinical trials to evaluate emerging compounds developed against novel targets or involving new mechanisms. Our goal is to understand the fundamental science as well as the condition to be treated, so that we can determine a way to evaluate whether the molecule is working as designed and having the anticipated effect.

We work extensively with biomarkers and a range of imaging techniques. We seek to develop clinical platforms and diagnostic technologies that will allow us to examine the biological activity and safety of novel compounds when they are introduced into people. These new translational models provide a window into human physiology, generating fresh scientific insight and stimulating further experimental questions.

Above all, it is gratifying to participate directly in the development of promising new treatments. But we were surprised to discover that our ability to recognize that a

\section{"Fundamentally, our work is team-based, and collaboration is absolutely required for success."}

compound is not working can be equally important. Across the drug industry, about $90 \%$ of compounds that enter clinical testing will fail; about a third of this attrition reflects a lack of efficacy. So the ability to make this determination early, and to divert resources into the design of better compounds rather than into additional clinical investigation of a flawed molecule, will ultimately increase the chances of bringing forward a truly useful drug.

Much of the excitement and satisfaction in our division comes from working with colleagues in many different regions of the organization, involved in every operational area, from basic discovery and medicinal chemistry through to manufacturing and marketing. Fundamentally, our work is team-based, and collaboration is absolutely required for success.

Of particular value to us are our relationships with colleagues in academia. Often, these are long-standing connections; but through our work in the experimental medicine division, we are increasingly meeting, discussing science and establishing collaborations with many researchers from around the world. These interactions broaden our perspective, emphasize the complexity of our shared challenges and highlight the importance of collaborative approaches.

Our fondest ambition is that the foundation we've developed will help catalyse the evolution of translational research into an integrated scientific discipline. We have established an infrastructure that can provide an expansive range of profiling and imaging platforms to our clinical investigators - capabilities that tend not to be present in most academic research environments. We are eager to contribute both our experience and our technology, and look forward to developing relationships with university investigators who share our passion for translational science.

Leaving academia was a difficult decision for both of us. But it is comforting to feel that in experimental medicine we have found an intellectual sanctuary where our vision of translational research is valued, our enthusiasm shared, our skills developed and our aspirations nurtured. After years of searching, it's nice to finally feel at home.

David Shaywitz and Hayes Dansky are associate directors, Department of Experimental Medicine, Merck Research Labs, Rahway, New Jersey, USA. 